\title{
REMARKS ON $\delta$-OPEN SETS INDUCED BY ENLARGEMENTS OF GENERALIZED TOPOLOGIES
}

\author{
Young Key Kim¹, Won Keun Min² ${ }^{2}$ \\ ${ }^{1}$ Department of Mathematics \\ MyongJi University \\ Yongin, 449-728, KOREA \\ ${ }^{2}$ Department of Mathematics \\ Kangwon National University \\ Chuncheon, 200-701, KOREA
}

\begin{abstract}
The concepts of $\kappa_{\mu}$-open set and enlargement $\kappa$ of a generalized topology $\mu$ were introduced by Császár [5]. In this paper, we introduce the concept of $\delta_{\mu}$-open set induced by an enlargement $\kappa$ of a generalized topology $\mu$, and we study some basic properties for the set. Moreover, we establish the relationships among $\kappa_{\mu}$-open sets, weak $\kappa_{\mu}$-open [6] and $\delta_{\kappa}$-open.
\end{abstract}

AMS Subject Classification: 54A05, 54B10, 54C10, 54D30

Key Words: generalized topology, enlargement, $\kappa_{\mu}$-open, $\kappa_{\mu}$-regular open, weak $\kappa_{\mu}$-open, $\delta_{\kappa}$-open

\section{Introduction}

Let $X$ be a nonempty set and $\exp (X)$ be the power set of $X$. Then $\mu \subseteq \exp (X)$ is called a generalized topology (briefly GT) [2] on $X$ iff $\emptyset \in \mu$ and $G_{i} \in \mu$ for $i \in I \neq \emptyset$ implies $G=\cup_{i \in I} G_{i} \in \mu$. We call the pair $(X, \mu)$ a generalized topological space (briefly GTS) on $X$. The elements of $\mu$ are called $\mu$-open $[1,2]$ sets and the complements are called $\mu$-closed sets. We call a GTS $X$ is strong if $X \in \mu[4]$. The generalized-closure of a subset $S$ of $X$, denoted by $c_{\mu}(S)$, is

Received: $\quad$ November 18, 2016

Revised: January 10, 2017

Published: January 24, 2017

(C) 2017 Academic Publications, Ltd. url: www.acadpubl.eu

${ }^{\S}$ Correspondence author 
the intersection of generalized closed sets including $S$. And the interior of $S$, denoted by $i_{\mu}(S)$, the union of generalized open sets included in $S$.

Let us define $\delta(\mu)=\delta \subseteq \exp (X)$ by $A \in \delta$ iff $A \subseteq X$ and, if $x \in A$, then there is a $\mu$-closed set $Q$ such that $x \in i_{\mu} Q \subseteq A$ [4]. $A$ is said to be $\mu r$-open (resp., $\mu r$-closed) [4] if $A=i_{\mu}\left(c_{\mu}(A)\right)$ (resp., $A=c_{\mu}\left(i_{\mu}(A)\right)$ ).

For a GT $\mu$ on $X$, a mapping $\kappa: \mu \rightarrow \exp X$ is called an enlargement [5] on $X$ if $M \subseteq \kappa(M)$ whenever $M \in \mu$. Let us say that a subset $A \subseteq X$ is $\kappa_{\mu}$-open [5] iff $x \in A$ implies the existence of a $\mu$-open set $M$ such that $x \in M$ and $\kappa(M) \subseteq A$. The collection $\kappa_{\mu}$ of all $\kappa_{\mu}$-open sets is a GT on $X$ and $\kappa_{\mu} \subseteq \mu[5]$. Let us say that a subset $A \subseteq X$ is $\kappa_{\mu}$-closed [5] iff $X-A$ is $\kappa_{\mu}$-open.

\section{Main Results}

Let $\mu$ be a GT on $X$ and $\kappa: \mu \rightarrow \exp X$ an enlargement of $\mu$. For $M \in \mu, M$ is said to be $\kappa$-regular open [6] if $i_{\mu}(\kappa(M))=M$. The complement of $\kappa$-regular open set is called a $\kappa$-regular closed set.

Definition 2.1. Let $(X, \mu)$ be a GTS, and let $\kappa$ be an enlargement of $\mu$ and $A \subseteq X$. Then $A$ is said to be $\delta_{\kappa}$-open if for each $x \in A$, there exists a $\kappa$-regular open set $G$ such that $x \in G \subseteq A$. The complement of a $\delta_{\kappa}$-open set is called a $\delta_{\kappa}$-closed set. We will denote $\delta_{\kappa}=\left\{A \subseteq X: A\right.$ is $\delta_{\kappa}$-open $\}$.

Remark 2.2. Let $\mu$ be a GT on $X$ and $\kappa: \mu \rightarrow \exp X$ an enlargement of $\mu$. Let us say that a subset $A \subseteq X$ is a weak $\kappa_{\mu}$-open (briefly w $\kappa_{\mu}$-open) set [6] iff $x \in A$ implies the existence of a $\mu$-open set $M$ such that $x \in M$ and $i_{\mu}(\kappa(M)) \subseteq A$. Then the collection $w \kappa_{\mu}$ of all weak $\kappa_{\mu}$-open sets is a GT on $X$ and $\kappa_{\mu} \subseteq w \kappa_{\mu} \subseteq \mu$. Furthermore, we can show easily that $\delta_{\kappa} \subseteq w \kappa_{\mu}$ and the notions of $\kappa_{\mu}$-open sets and $\delta_{\kappa}$-open sets are independent. So, we have the following diagram:

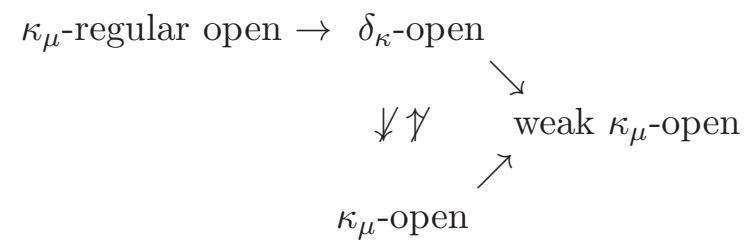

The converses are not true always as shown in the next examples:

Example 2.3. Let $X=\{a, b, c, d, e\}$ and a generalized topology $\mu=$ $\{\emptyset,\{a\}\{b, c\},\{a, b, c\},\{a, b, c, d\}\}$ on $X$. Let us consider an enlargement $\kappa$ : $\mu \rightarrow \exp X$ defined as the following: 


$$
\kappa(M)= \begin{cases}M \cup\{d\}, & \text { if } a \in M, \\ M \cup\{a\}, & \text { if } a \notin M .\end{cases}
$$

Then $\kappa$ is an enlargement. Note that:

$$
\begin{aligned}
& \kappa(\{a\})=\{a, d\} ; \kappa(\{b, c\})=\{a, b, c\} ; \kappa(\{a, b, c\})=\{a, b, c, d\} \\
& i \kappa(\{a\})=\{a\} ; i \kappa(\{b, c\})=\{a, b, c\} ; i \kappa(\{a, b, c\})=\{a, b, c, d\} .
\end{aligned}
$$

For $A=\{a, b, c\}, A$ is weak $\kappa_{\mu}$-open but not $\delta_{\kappa}$-open.

Example 2.4. Let $X=\{a, b, c, d\}$ and a generalized topology $\mu=\{\emptyset,\{a\}$ $\{b, c\},\{a, b, c\}, X\}$ on $X$. Let us consider an enlargement $\kappa: \mu \rightarrow \exp X$ defined as the following:

$$
\kappa(M)= \begin{cases}M \cup\{d\}, & \text { if } a, b \in M, \\ M \cup\{c\}, & \text { otherwise. }\end{cases}
$$

Then $\kappa$ is an enlargement. Note that:

$$
\begin{aligned}
& \kappa(\{a\})=\{a, c\} ; \kappa(\{b, c\})=\{b, c\} ; \kappa(\{a, b, c\})=X \\
& i \kappa(\{a\})=\{a\} ; i \kappa(\{b, c\})=\{b, c\} ; i \kappa(\{a, b, c\})=X .
\end{aligned}
$$

For $A=\{a\}, A$ is $\delta_{\kappa}$-open but not $\kappa_{\mu}$-open. For $B=\{a, b, c\}, B$ is $\delta_{\kappa}$-open but not $\kappa_{\mu}$-regular open.

Example 2.5. Let $X=\{a, b, c, d\}$ and a generalized topology $\mu=$ $\{\emptyset,\{a, b\}\{b, c\},\{a, b, c\}, X\}$ on $X$. Let us consider an enlargement $\kappa: \mu \rightarrow$ $\exp X$ defined as the following:

$$
\kappa(M)= \begin{cases}M \cup\{d\}, & \text { if } a, c \in M, \\ M \cup\{c\}, & \text { otherwise. }\end{cases}
$$

Then $\kappa$ is an enlargement. Note that:

$$
\begin{gathered}
\kappa(\{a, b\})=\{a, b, c\} ; \kappa(\{b, c\})=\{b, c\} ; \kappa(\{a, b, c\})=X ; \\
i \kappa(\{a, b\})=\{a, b, c\} ; i \kappa(\{b, c\})=\{b, c\} ; i \kappa(\{a, b, c\})=X .
\end{gathered}
$$

For $A=\{a, b, c\}, A$ is $\kappa_{\mu}$-open but not $\delta_{\kappa}$-open.

Let $X$ be a nonempty set and $s \subseteq 2^{X}$. Then $s$ is called a $\sigma$-structure [7] on $X$ if for $i \in I \neq \emptyset, U_{i} \in s$ implies $\cup_{i \in I} U_{i} \in s$. The elements of $s$ are called $\sigma$-open sets and the complements are called $\sigma$-closed sets. 
Theorem 2.6. Let $(X, \mu)$ be a GTS, and let $\kappa$ be an enlargement of $\mu$. Then $\delta_{\kappa}$ is a $\sigma$-structure.

Proof. Let $Q \in \delta_{\kappa}$ and $x \in Q$. Then there exists a $\kappa$-regular open set $G_{x}$ such that $x \in G_{x} \subseteq Q$ and so $\cup_{x \in Q} G_{x}=Q$.

In general, $\delta_{\kappa}$ is not a generalized topology in the sense of Császár as shown in the next example:

Example 2.7. Let $X=\{a, b, c, d\}$. Consider generalized topologies $\mu=$ $\{\emptyset,\{a\}\{b, c\},\{a, b, c\}\}$ on $X$. Let us consider an enlargements $\kappa: \mu \rightarrow \exp X$ defined as the following: $\kappa G=G \cup\{a\}$ for $G \in \mu$. Then for $G=\emptyset$,

$$
\kappa(G)=\{a\}, \quad i \kappa(G)=i\{a\}=\{a\} \neq \emptyset .
$$

Hence, the empty set $\emptyset$ is not $\kappa$-regular open.

Let $\mu$ be a GT on $X$ and $\kappa_{\mu}$ an enlargement of $\mu$. The enlargement $\kappa$ is said to be ordinary [6] if $\kappa \subseteq c_{\mu}$ for $M \in \mu$.

Theorem 2.8. Let $\mu$ be a GT on $X$ and $\kappa_{\mu}$ an enlargement of $\mu$. If $\kappa$ is ordinary, then $\delta_{\kappa}$ is a generalized topology.

Proof. From $\emptyset=i_{\mu}\left(c_{\mu}(\emptyset)\right)$, it follows $\emptyset \subseteq i_{\mu}(\kappa(\emptyset)) \subseteq i_{\mu}\left(c_{\mu}(\emptyset)\right)$. Hence $\emptyset$ is $\kappa$-regular open. Hence from Theorem 2.6, $\delta_{\kappa}$ is a generalized topology.

Theorem 2.9. Let $(X, \mu)$ be a GTS, and let $\kappa$ be an enlargement of $\mu$. If $\kappa$ is quasi-idempotent and monotonic, then for every $\mu$-open set $M, i_{\mu}(\kappa(M))$ is $\delta_{\kappa}$-open.

Proof. From hypothesis and $M \subseteq i_{\mu}(\kappa(M))$, it follows

$$
i_{\mu}(\kappa(M)) \subseteq i_{\mu}\left(\kappa\left(i_{\mu}(\kappa(M))\right)\right) \subseteq i_{\mu}(\kappa(M)) .
$$

So $i_{\mu}\left(\kappa\left(i_{\mu}(\kappa(M))\right)\right)=i_{\mu}(\kappa(M))$. So, $i_{\mu}(\kappa(M))$ is $\kappa$-regular open for every $\mu$ open set $M$, and $i_{\mu}(\kappa(M))$ is $\delta_{\kappa}$-open.

Theorem 2.10. Let $(X, \mu)$ be a GTS, and let $\kappa$ be a monotonic enlargement of $\mu$. If $\mu$ is strong, then $\delta_{\kappa}$ is strong.

Proof. Since $\mu$ is strong and $\kappa$ is a monotonic enlargement of $\mu, \kappa(X)=X$ and $X$ is $\mu$-open. So, $X$ is $\kappa$-regular open. Hence, $\delta_{\kappa}$ is strong. 
A GT $\mu$ is called a quasi-topology (briefly QT) [3] on $X$ if it satisfies the following: For $U_{1}, U_{2} \in \mu, U_{1} \cap U_{2} \in \mu$.

Theorem 2.11. Let $(X, \mu)$ be a GTS, and let $\kappa$ be an enlargement of $\mu$. If $\mu$ is $Q T$ and $\kappa$ is monotonic and ordinary, then $\delta_{\kappa}$ is $Q T$.

Proof. First, since $\kappa$ is ordinary, $\delta_{\kappa}$ is GT. In order to complete the proof, it is sufficient to show that $U_{1} \cap U_{2}$ is $\kappa$-regular open, for $\kappa$-regular open sets $U_{1}$ and $U_{2}$. For $\kappa$-regular open sets $U_{1}$ and $U_{2}$, since $\mu$ is QT, $U_{1} \cap U_{2} \in \mu$. Since $\kappa$ is monotonic, $U_{1} \cap U_{2}=i_{\mu}\left(U_{1} \cap U_{2}\right) \subseteq i_{\mu}\left(\kappa\left(U_{1} \cap U_{2}\right)\right) \subseteq i_{\mu}\left(\kappa\left(U_{1}\right)\right) \cap i_{\mu}\left(\kappa\left(U_{2}\right)\right)=$ $U_{1} \cap U_{2}$. Hence, $U_{1} \cap U_{2}$ is $\kappa$-regular open.

Corollary 2.12. Let $(X, \mu)$ be a GTS, and let $\kappa$ be an enlargement of $\mu$. If $\mu$ is a topology and if $\kappa$ is monotonic and ordinary, then $\delta_{\kappa}$ is a topology.

Proof. Since a topology is a strong GT and a QT, it follows from Theorem 2.10 and Theorem 2.11.

Definition 2.13. The $\delta_{\kappa}$-closure of a subset $A$ of $X$, denoted by $c_{\delta_{\kappa}}(A)$, is the intersection of $\delta_{\kappa}$-closed sets including $A$. And the $\delta_{\kappa}$-interior of $A$, denoted by $i_{\delta_{\kappa}}(A)$, the union of $\delta_{\kappa}$-open sets included in $A$.

Theorem 2.14. Let $\mu$ be a $G T$ on $X, A \subseteq X$ and $\kappa$ an enlargement of $\mu$. Then

(1) $x \in c_{\delta_{\kappa}}(A)$ if and only if for every $\kappa$-regular open set $G$ containing $x$, $G \cap A \neq \emptyset$.

(2) $x \in i_{\delta_{\kappa}}(A)$ if and only if there exists a $\kappa$-regula open set $M$ containing $x$ such that $M \subseteq A$.

Proof. Obvious.

Theorem 2.15. Let $\mu$ be a GT on $X$ and $\kappa$ an ordinary enlargement of $\mu$. Then the following hold.

(1) If $A \subseteq B \subseteq X$, then $c_{\delta_{\kappa}}(A) \subseteq c_{\delta_{\kappa}}(B)$ and $i_{\delta_{\kappa}}(A) \subseteq i_{\delta_{\kappa}}(B)$.

(2) For $A \subseteq X, i_{\delta_{\kappa}}(A)$ is $\mu$-open.

Proof. It is obvious since $\delta_{\kappa}$ is a GT on $X$.

Theorem 2.16. Let $\mu$ be a $G T$ on $X, A \subseteq X$ and $\kappa$ an enlargement of $\mu$. If $\kappa$ is quasi-idempotent and monotonic, then the following hold.

(1) $x \in c_{\delta_{\kappa}}(A)$ if and only if $x \in M$ and $M \in \mu$ implies $i_{\mu}(\kappa(M)) \cap A \neq \emptyset$.

(2) $x \in i_{\delta_{\kappa}}(A)$ if and only if there exists a $\mu$-open set $M$ containing $x$ such that $i_{\mu}(\kappa(M)) \subseteq A$. 
Proof. (1) For each $\mu$-open set $M$ containing $x$, by Theorem $2.9, i_{\mu}(\kappa(M))$ is a $\kappa$-regular open set containing $x$. Since $x \in c_{\delta_{\kappa}}(A)$, we have $i_{\mu}(\kappa(M)) \cap A \neq \emptyset$.

For the converse, let $Q$ be any $\delta_{\kappa}$-open set containing $x$. Then there is a $\kappa$-regular open set $G$ such that $x \in G \subseteq Q$. Since $G$ is a $\mu$-open set of $x$, from hypothesis, it follows $\emptyset \neq i_{\mu}(\kappa(G)) \cap A=G \cap A \subseteq Q \cap A$. Hence $x \in c_{\delta_{\kappa}}(A)$.

(2) Let $x \in i_{\delta_{\kappa}}(A)$. Then there is a $\delta_{\kappa}$-open set $Q$ containing $x$ such that $x \in Q \subseteq A$. Moreover, there is a $\kappa$-regular open set $M$ such that $x \in M \subseteq Q$. Since $M$ is a $\mu$-open set of $x$ and $i_{\mu}(\kappa(M))=M, i_{\mu}(\kappa(M)) \subseteq A$.

For the converse, suppose that there exists a $\mu$-open set $M$ containing $x$ such that $i_{\mu}(\kappa(M)) \subseteq A$. Then since $i_{\mu}(\kappa(M))$ is $\kappa$-regular open, $x \in i_{\delta_{\kappa}}(A)$.

Theorem 2.17. Let $\mu$ be a GT on $X, A \subseteq X$ and $\kappa$ an enlargement of $\mu$. If $\kappa$ is quasi-idempotent, monotonic and ordinary, then for any $M \in \mu$, $x \in c_{\delta_{\kappa}}(M)$ if and only if $x \in G$ and $G \in \mu$ implies $\kappa(G) \cap M \neq \emptyset$.

Proof. Suppose that $x \in G$ and $G \in \mu$ implies $\kappa(G) \cap M \neq \emptyset$. Then for any $\mu$-open set $G$ containing $x$, since $\kappa$ is ordinary, $\emptyset \neq \kappa(G) \cap M \subseteq c_{\mu}(G) \cap M$. Since $c_{\mu}(G) \cap M \neq \emptyset$, we have $G \cap M \neq \emptyset$ and $\emptyset \neq G \cap M \subseteq i_{\mu}(\kappa(G)) \cap M$. Hence, $x \in c_{\delta_{\kappa}}(M)$.

Conversely, let $x \in c_{\delta_{\kappa}}(M)$ for $M \in \mu$. Then for any $\mu$-open set $G$ containing $x$, from Theorem 2.15, $\emptyset \neq i_{\mu}(\kappa(G)) \cap M \subseteq \kappa(G) \cap M$. So the statement is obtained.

\section{Acknowledgments}

This work was supported by 2016 Research Fund of Myongji University.

\section{References}

[1] Á. Császár, Generalized open sets, Acta Math. Hungar., 75 (1997), 65-87.

[2] Á. Császár, Generalized topology, generalized continuity, Acta Math. Hungar., 96 (2002), 351-357.

[3] Á. Császár, Further remarks on the formular for $\gamma$-interior, Acta Math. Hungar., 113 (2006), 325-332.

[4] Á. Császár, $\delta$ - and $\theta$-modificatons of generalized topologies, Acta Math. Hungar., 120, No. 3 (2008), 275-279. 
[5] Á. Császár, Enlargements and generalized topologies, Acta Math. Hungar., 120, No. 4 (2008), 351-354.

[6] Y. K. Kim and W. K. Min, Further remarks on Enlargements of generalized topologies, Acta Math. Hungar., 135, No. 1 (2012), 184191.

[7] Y. K. Kim and W. K. Min, $\sigma$-structures and quasi-enlarging operations, International Journal of Pure and Applied Mathematics, 86, No. 5 (2013), 791-798. 
